\title{
Atlas de la minería metálica en México
}

\author{
Sol Pérez Jiménez* \\ Recibido el 23 de noviembre de 2015; aceptado el 11 de julio de 2016
}

\begin{abstract}
Mining is increasingly important to analyze both its environmental and social impacts. However, the information on the territorial deployment of this activity is not of public domain. In response to this problem, we present the full Mapping metallic mining in Mexico. By using Geographic Information Systems we elaborated an Atlas, in which is shown the spatial deployment of this activity in all the Country. Mainly we present the list of projects and mining concessions which are inside of important areas for water resources or under some category of environmental protection and indigenous territories.
\end{abstract}

Key words: Cartography, Mining, Mexico, Environmental Impact.

\section{Resumo}

A mineração é uma atividade cada vez mais importante de se analisar, tanto por seus impactos ambientais como pelos sociais. Entretanto, a informação sobre o desenvolvimento territorial desta atividade não é de domínio público. Como resposta a este problema, neste trabalho apresentamos a cartografia completa sobre mineração metálica no México. Graças ao uso de Sistemas de Informação Geográfica foi elaborado um Atlas, no qual se mostra o desenvolvimento espacial desta atividade ao longo do território nacional. Apresentamos principalmente a relação de projetos e concessões de mineração em zonas importantes para recursos hídricos, relacionado a alguma categoria de proteção ambiental e territórios indígenas.

Palavras chave: Cartografia, mineração, México, impacto ambiental.

\section{Resumen}

La minería es una actividad cada vez más importante de analizar, tanto por sus impactos ambientales como sociales. Sin embargo, la información sobre el desplie-

* Universidad Nacional Autónoma de México, correo electrónico: solperezj@gmail.com 
gue territorial de esta actividad no es de dominio público. En respuesta a este problema, en el trabajo presentamos la cartografía completa sobre minería metálica en México. Gracias al uso de Sistemas de Información Geográfica se logró la realización de un Atlas, en el cual se muestra el despliegue espacial de esta actividad a lo largo del territorio nacional. Presentamos principalmente la relación de proyectos y concesiones mineras con zonas importantes para el recurso hídrico, bajo alguna categoría de protección ambiental y territorios indígenas.

Palabras clave: Cartografía, minería, México, impacto ambiental.

\section{Introducción}

Es importante profundizar en el tema de minería metálica en México, ya que sus impactos ambientales son inmensurables. En aras de la mega minería metalífera en México se está "transformando a las comunidades y sus paisajes; mercantilizando terrenos ancestrales; amenazando recursos naturales y las formas de vida que dependen de ellos y transformando un conjunto de prácticas culturales y políticas tradicionales que, históricamente, han moldeado el paisaje" (Bebbington: 2009: 4).

Sin embargo, pese a que existen una gran cantidad de estadísticas entorno al ámbito minero, aún existen "importantes problemas de transparencia sobre la gestión pública de las actividades mineras" (Centro de Análisis de Investigación -FUNDAR, 2013). A pesar de que tanto el derecho nacional como el internacional reconocen que las y los ciudadanos tienen el derecho a acceder a la información generada por entes públicos (Toronto Transit Commission - TTC, 2010), pues el papel de estos es servir a la ciudadanía y la información que crean y mantienen pertenece a ella, a menudo se imposibilita su acceso. En la Tabla 1, se muestran los principales problemas entorno al control de la información referente al rubro minero en México.

"La Cartografía Minera”, por ejemplo, es un documento público que comprende la localización exacta de todas las concesiones, asignaciones y reservas mineras en el territorio nacional. A pesar de que es posible consultar este documento en el Sistema Integral de Administración Minera (SIAM), la plataforma presenta diversos problemas en términos de accesibilidad (véase Tabla 1). El SIAM sólo permite visualizar, más no descargar, los polígonos que establecen los límites de las concesiones, asignaciones y reservas mineras. Esto dificulta su estudio, sobre todo cuando se busca conocer si se encuentran sobre Áreas Naturales Protegidas (ANP), núcleos agrarios, núcleos de población, cuencas hidrográficas, zonas arqueológicas, territorios indígenas, etc. Por otra parte, el artículo 66 de la Ley Federal de Derechos establece un cobro de $\$ 3,000.00$ (pesos mexicanos) por cada carta a escala 1:50,000 de la Cartografía Minera. De tal modo, para poder tener un mapa completo del territorio nacional, es necesario erogar $\$ 6,013,392.86$. Ahora bien, estos ma- 
pas se actualizan mensualmente, por lo cual los gastos por mantener un mapa actualizado ascienden a $\$ 72,160,714.32$ anuales.

Tabla 1

Problemas de acceso a la información sobre minería en México

\begin{tabular}{ll}
\hline \multicolumn{1}{c}{ Problema } & \multicolumn{1}{c}{ Tipo de información } \\
\hline Candados legales & $\begin{array}{l}\text { Información sobre la producción, beneficio y } \\
\text { destino de los minerales (extraídos) } \\
\text { Geología de los yacimientos y reservas de mineral } \\
\\
\text { Estados económicos (...) de empresas mineras y } \\
\text { metalúrgicas }\end{array}$ \\
Cobros exorbitantes por & Cartografía minera \\
información & Información sobre los yacimientos (SGM) \\
Accesibilidad a & La información sobre el sector no se encuentra concentrada \\
información & Las plataformas actualmente disponibles no \\
disponible & permiten un uso eficiente de la información pública \\
& La publicación de información en línea no es suficiente \\
& para garantizar el derecho al acceso a la información \\
\hline
\end{tabular}

Fuente: Elaboración propia con base a FUNDAR, 2013.

Por otro lado, la información oficial referente a los proyectos mineros en México es imprecisa y está dispersa. Si bien existen los Panoramas mineros por estado publicados por el mismo Servicio Geológico Mexicano, en éstos sólo vienen los proyectos en fase de operación. Para conocer los proyectos en exploración, desarrollo o suspensión, en el portal web de dicha dependencia están disponibles tablas en formato .pdf con listas de los proyectos. Desafortunadamente, en ninguno de los casos viene su ubicación geográfica.

El resultado es que a la fecha, ni los mapas sobre concesiones mineras ni los de proyectos mineros en México son de libre acceso. Ello ha dificultado un análisis real de la dimensión de esta actividad a nivel nacional. Si bien existen estudios académicos, éstos están elaborados con las estadísticas de la Secretaría de Economía (Koll-Hurtado, 2002; Sánchez-Salazar, 2010), no existen trabajos que aborden el despliegue territorial actual de la minería a nivel nacional en toda su complejidad, es decir, respecto a las concesiones y proyectos en todas sus etapas (operación, desarrollo, exploración y suspensión), tipo de metales extraídos, país de origen de capital así como su impacto a los recursos hídricos, la biodiversidad y pueblos indígenas. Es en ese sentido que el trabajo aquí presentado cobra relevancia. Pues, a partir de diversas fuentes de información oficiales, se elaboró tanto la cartografía sobre proyectos mineros como de las concesiones y se comparó con dichas variables. 


\section{Metodología}

Para el mapa de los proyectos mineros se utilizaron diferentes fuentes. El primer insumo fue la cartografía generada por Pérez-Jiménez (2012) para los proyectos mineros operando en México según los Panoramas Mineros (SGM, 2011). El segundo fue la lista de proyectos mineros en México de la Secretaría de Economía (SE, 2013). Y el tercero fue el portal de información Mexico Mining Center (MMC, 2014). Primero se actualizó la información sobre los proyectos mineros en operación para el año 2014. Posteriormente se prosiguió a buscar la ubicación geográfica (latitud, longitud) de cada proyecto minero en exploración, desarrollo o suspensión en base a sus manifestaciones de impacto ambiental. Dicha información se fue añadiendo a la tabla de la SE. La cual únicamente incluye los proyectos mineros operados por compañías extranjeras. Para verificar y ampliar la información, se comparó la tabla de la SE con la del MMC, que sí incluye los proyectos llevados a cabo por compañías mexicanas, los cuales también se ubicaron geográficamente. El resultado fue la cartografía completa de proyectos mineros en México.

El mapa de concesiones mineras se realizó con base al SIAM, que en su portal de internet muestra los polígonos de las concesiones mineras en México. Los cuales se digitalizaron manualmente en un Sistema de Información Geográfica (SIG) de libre acceso (QGIS).

Posteriormente se comparó dicha cartografía con la generada por la Comisión Nacional para el Conocimiento y Uso de la Biodiversidad (CONABIO), por la Comisión Nacional del Agua (CONAGUA) y por el Instituto Nacional de Estadística y Geografía (INEGI) a escala 1:1,000,000.

\section{Nueva geografía de la minería en México}

Algunos autores han descrito como, "la nueva geografía de la minería de tajo a cielo abierto en América Latina se extiende al México rural, con efectos drásticos sobre la tierra, el agua y otros recursos de subsistencia de las sociedades campesinas" (Garibay et al., 2011:2).

Esta nueva geografía envuelve la explotación de los minerales que ya no se encuentran concentrados en las venas y son imposibles de extraer por minería subterránea. Los minerales dispersos con bajas concentraciones en grandes áreas requieren ahora minería a cielo abierto (Mudd, 2007). En otras palabras, "la nueva geografía de la minería en América Latina se ha movido de venas abiertas a tajos abiertos" (Bebbington, 2009:7). Las compañías mineras se presentan a ellas mismas como agentes responsables de cambio progresivo y, los gobiernos casi invariablemente dan soporte a la minería para acceder a divisas, empleo y los ingresos fiscales que genera (Bebbington, Bury et al., 2008). Sin embargo, la dinámica de extracción de recursos todavía se compone de paisajes frágiles sometidos a la per- 
turbación del medioambiente y la destrucción de otros modos de vida para la acumulación de beneficios en otros espacios (Ibídem.).

Si bien desde las ciencias sociales se estudian cada vez más las protestas sociales en las áreas urbanas y rurales afectadas por la minería (Wallenius, 2010). Ninguno de los autores mencionados ha cartografiado esta Nueva geografía de la minería de la que tanto se habla, no al menos para el caso mexicano. La mayoría se dedican a describir "las asimetrías de poder entre comunidades y empresas mineras, y la influencia del Estado, especialmente en la elaboración y puesta en práctica de los contratos entre la comunidad y la corporación" (Bebbington and Bury, 2008; O'Faircheallaigh, 2010; Wallenius, 2010; Garibay et al., 2011; Tetreault, 2012). Y a pesar de que existen iniciativas interesantes para elaborar cartografía sobre el tema en Argentina, Guatemala, Perú y Chile (Svampa, 2008; Gudynas, 2011; Observatorio Latinoamericano de Conflictos Mineros - OCMAL, 2013), en términos geográficos no se profundizan más allá de la localización geográfica de los proyectos. Lo cual, si bien es útil, no es suficiente para entender el despliegue territorial de la minería.

En cambio, por el carácter geográfico del trabajo entendemos que espacializar no sólo es localizar. El análisis espacial permite, entre otros, encontrar $-\mathrm{O}$ nopatrones y procesos de fenómenos naturales y/o sociales en el espacio geográfico. A través de este tipo de análisis se pueden encontrar relaciones entre los fenómenos estudiados; sin embargo aquí es importante aclarar que por ser este un trabajo único en su tipo, por ahora nos dedicamos a analizar el despliegue territorial de la minería en términos únicamente de coincidencia espacial y no de causalidad.

En México existen 1,189 proyectos mineros. En el Mapa 1 se muestra como 880 están en etapa de exploración; 197 en etapa de producción, 67 en suspensión y 45 en desarrollo, véase Gráfica 1. Sobre el tipo de metal a extraer en cada proyecto, en el Mapa 2 se puede observar como 704 proyectos en México están dirigidos exclusivamente a la extracción de metales preciosos. Así mismo, la Gráfica 2 ejemplifica como, si consideramos que la combinación de estos con un metal base también tiene como objetivo fundamental la remoción de oro y plata del subsuelo, podemos afirmar que, el $68 \%$ de los proyectos mineros en México se centran en la extracción de oro y plata. Únicamente el $12 \%$ de los proyectos (147 de 1189) tiene por objetivo producir metales básicos o industriales. Mientras que el 20\% restante prioriza tanto los metales preciosos como los industriales.

Esto último es importante porque en el debate público sobre la minería es común escuchar argumentos tan planos como ¿qué no usan computadoras, autos y celulares estás gentes?, ¿creen que podemos vivir sin metales? Si bien es un hecho que necesitamos metales, pero ¿hace falta toda la cantidad y variedad de minerales que hoy se extraen?, ¿necesitamos seguir extrayendo oro? En el año 2014, por ejemplo, la demanda mundial de oro para cubrir usos tecnológicos e industriales fue 
de 463 toneladas. El resto fue utilizado para hacer lingotes, monedas o joyería. Eso significa que el $89 \%$ del oro es utilizado para la acumulación de capital o hacer anillos y aretes (WWC, 2014). Así mismo, los usos industriales y odontológicos del oro podrían ser satisfechos durante 66 años con las "reservas" de bancos centrales y Fondo Monetario Internacional (FMI), o 354 años con el total de oro ya extraído (Giraud, 2012).

Sobre la retribución de esta industria a las cuentas nacionales, señalemos que en algunos países de Latinoamérica los derechos de minería se pagan por las ventas de los productos, mientras que en México, hasta el año 2014, los mismos se generaban principalmente por el número de hectáreas concesionadas. Lo cual significa un notable beneficio económico para las empresas que realizan estas actividades, puesto que en territorio nacional el cobro por hectárea concesionada varía entre $\$ 5.00 \mathrm{y}$ \$111.00 (Gonzáles, 2012). Por ejemplo, de acuerdo con Peláez (2013), al comparar el valor de la producción con el pago de derechos en los últimos años se puede observar la enorme diferencia entre el valor de los metales extraídos y los módicos pagos que las compañías hacen para tener derecho a dicha extracción, véase Tabla 2.

Tabla 2

Comparativo del valor de la producción minero-metalúrgica con los ingresos por derechos de concesiones mineras (miles de pesos mexicanos)

\begin{tabular}{rrrrr}
\hline Año & Tonelada & $\begin{array}{r}\text { Valor dela } \\
\text { producción }\end{array}$ & Derechos & Diferencia \\
\hline 2005 & $40,697,037$ & $52,131,714.8$ & $394,775.9$ & $51,736,938.9$ \\
2006 & $43,967,590$ & $78,561,236.3$ & $322,696.6$ & $78,238,539.7$ \\
2007 & $45,813,099$ & $90,271,718.8$ & $421,572.9$ & $89,850,145.9$ \\
2008 & $45,910,031$ & $94,018,295.4$ & $1,667,042.8$ & $92,351,252.6$ \\
2009 & $43,093,364$ & $94,816,874.6$ & $1,781,762.7$ & $93,035,111.9$ \\
2010 & $47,017,649$ & $142,622,589.4$ & $1,955,566.5$ & $140,667,022.9$ \\
\hline Total & $266,498,770$ & $552,422,429.3$ & $6,543,417.4$ & $545,879,011.9$ \\
\hline
\end{tabular}

Fuente: Volumen y valor de la producción minero-metalúrgica, emitido por la Dirección General Minería (DGM) del periodo 2005 a 2010.

Como se puede observar en el mapa 3, de los 1189 proyectos mineros en México, Canadá controla 810, Estados Unidos de América 151, México 131, China 39, Australia 26, Reino unido 8, Perú 7, Argentina-Italia/India 6, Corea 3, Japón/México 2, India 2 y Chile, Italia, Suiza y Bélgica 1 (respectivamente). Así, el $89 \%$ de la minería en México es manejada por capital extranjero, el 11\% restante lo operan empresas mineras mexicanas, véase Gráfica 3. 


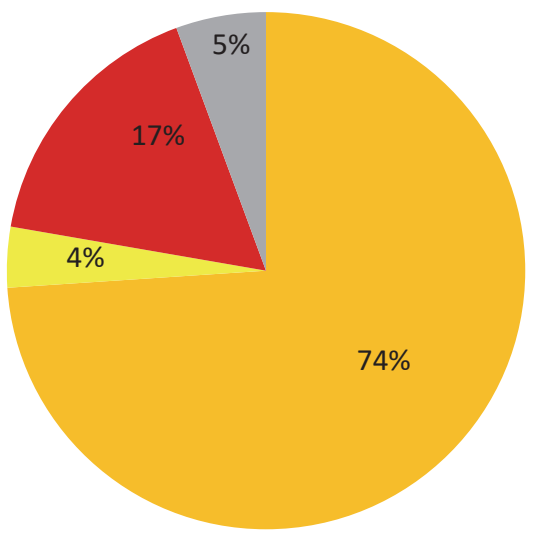

Gráfica 1. Proyectos mineros en México por etapa.

Fuente: SGM, 2011; SE, 2014; MMC, 2014.

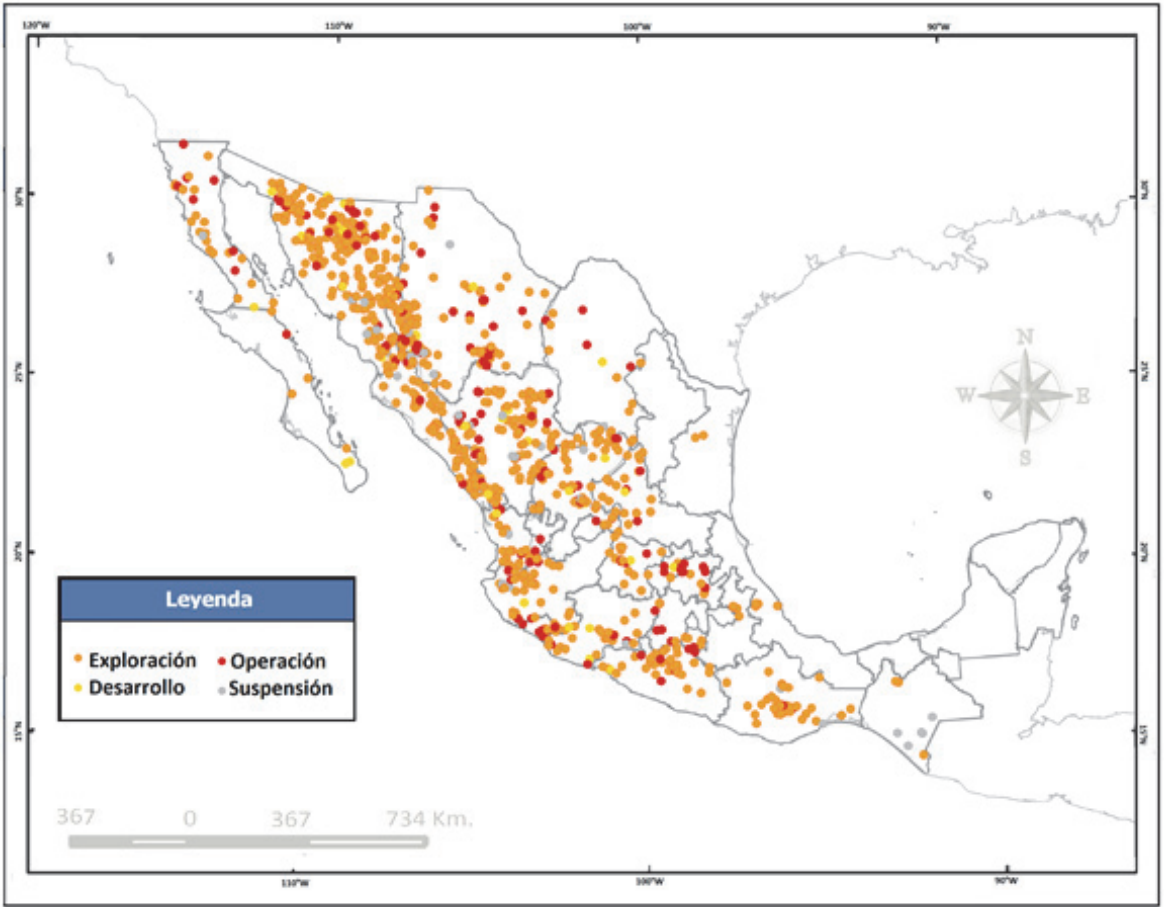

Mapa 1. Proyectos mineros en México por etapa.

Fuente: SGM, 2011; SE, 2014; MMC, 2014. 


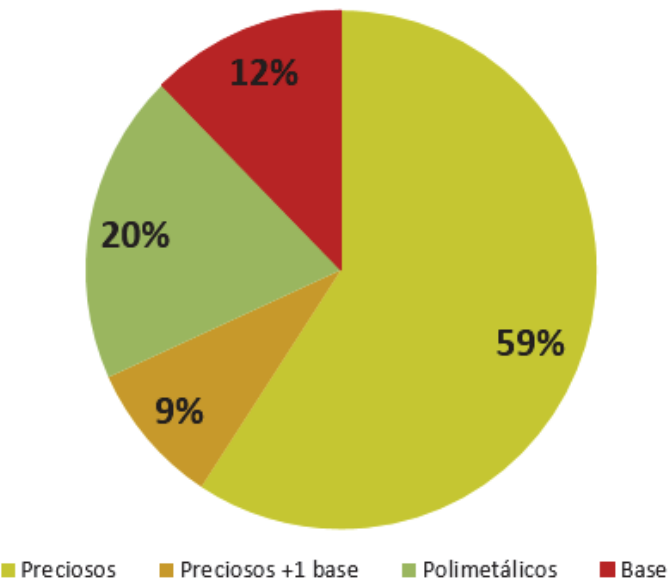

Gráfica 2. Proyectos mineros en Méxcio por tipo de mineral (2013). Fuente: SGM, 2011; SE, 2014.

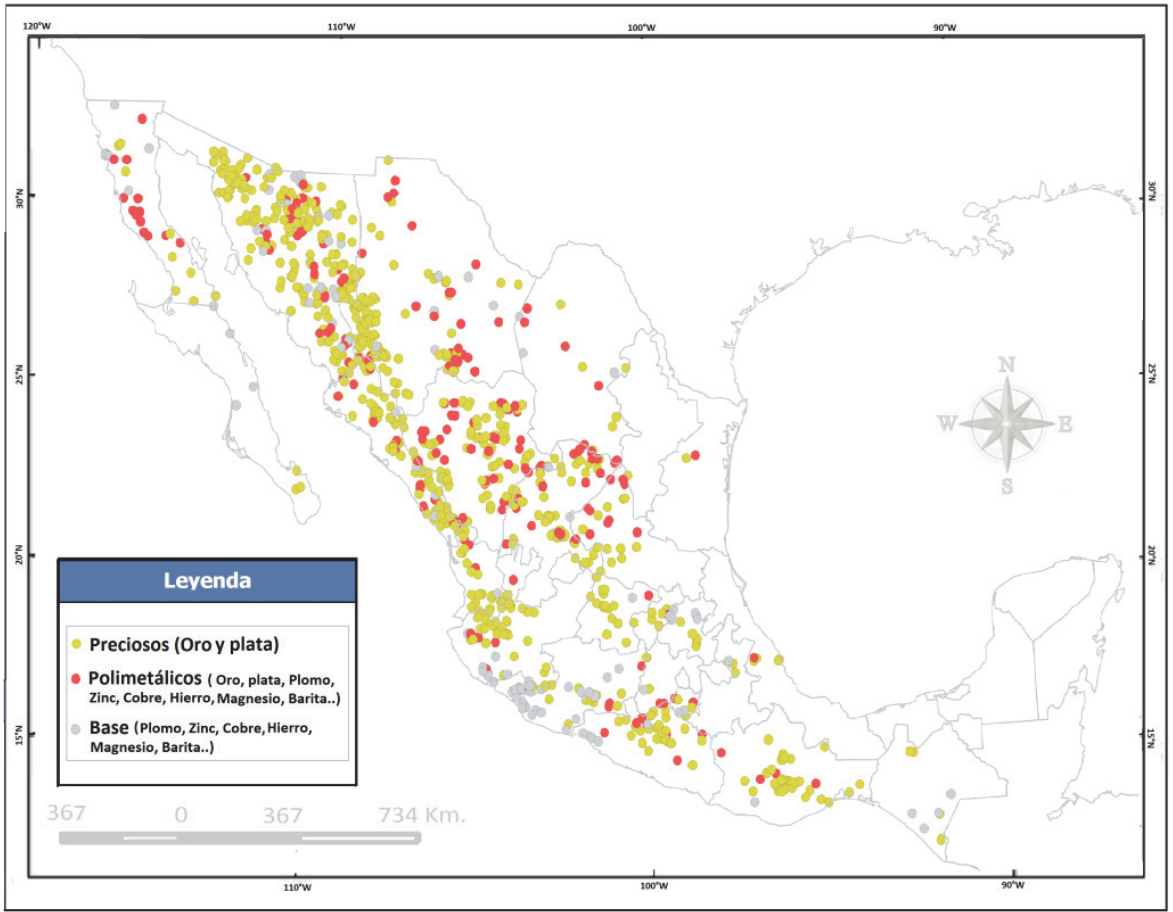

Mapa 2. Proyectos mineros en México por un tipo de mineral.

Fuente: SGM, 2011; SE, 2014; MMC, 2014. 


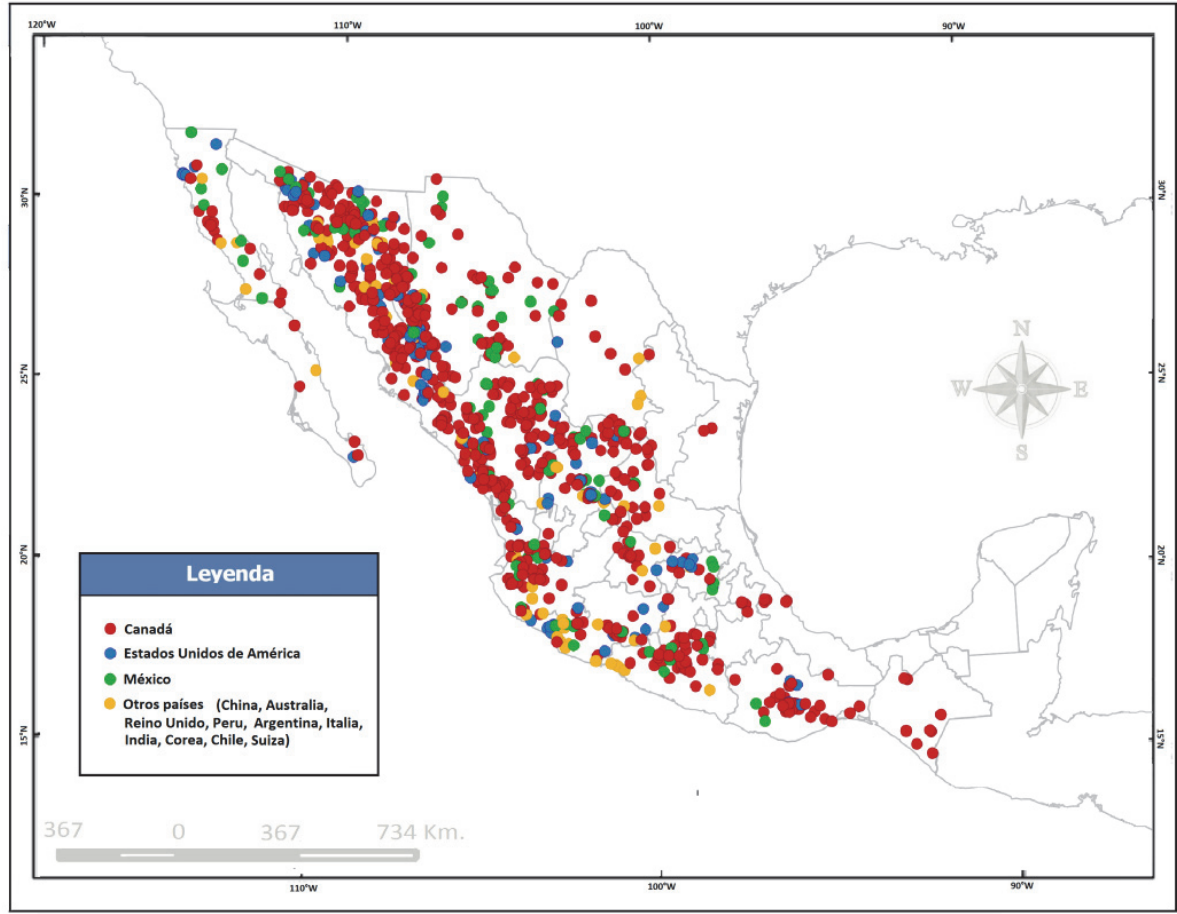

Mapa 3. Proyectos mineros en México por país de origen de capital. Fuente: SGM, 2011; SE, 2014; MMC, 2014.

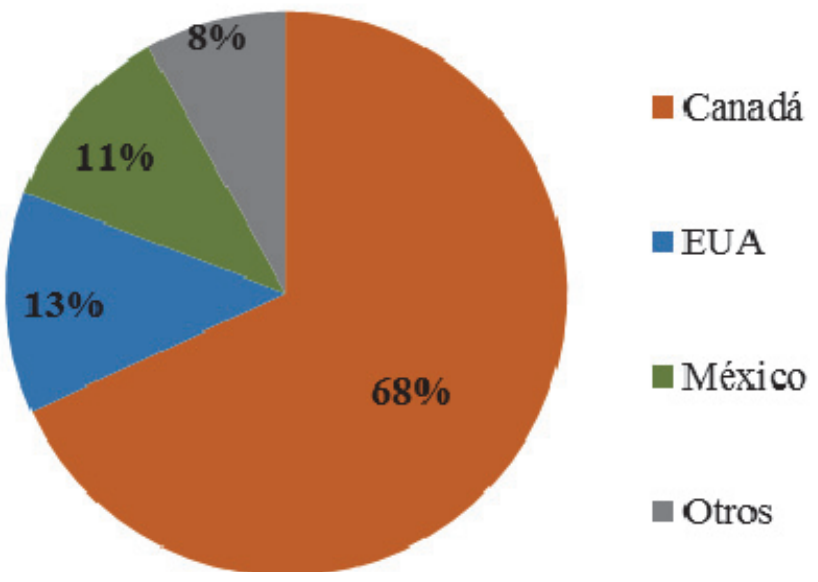

Gráfica 3. Proyectos mineros en México por país de origen del capital. Fuente: SGM, 2011; SE, 2014. 


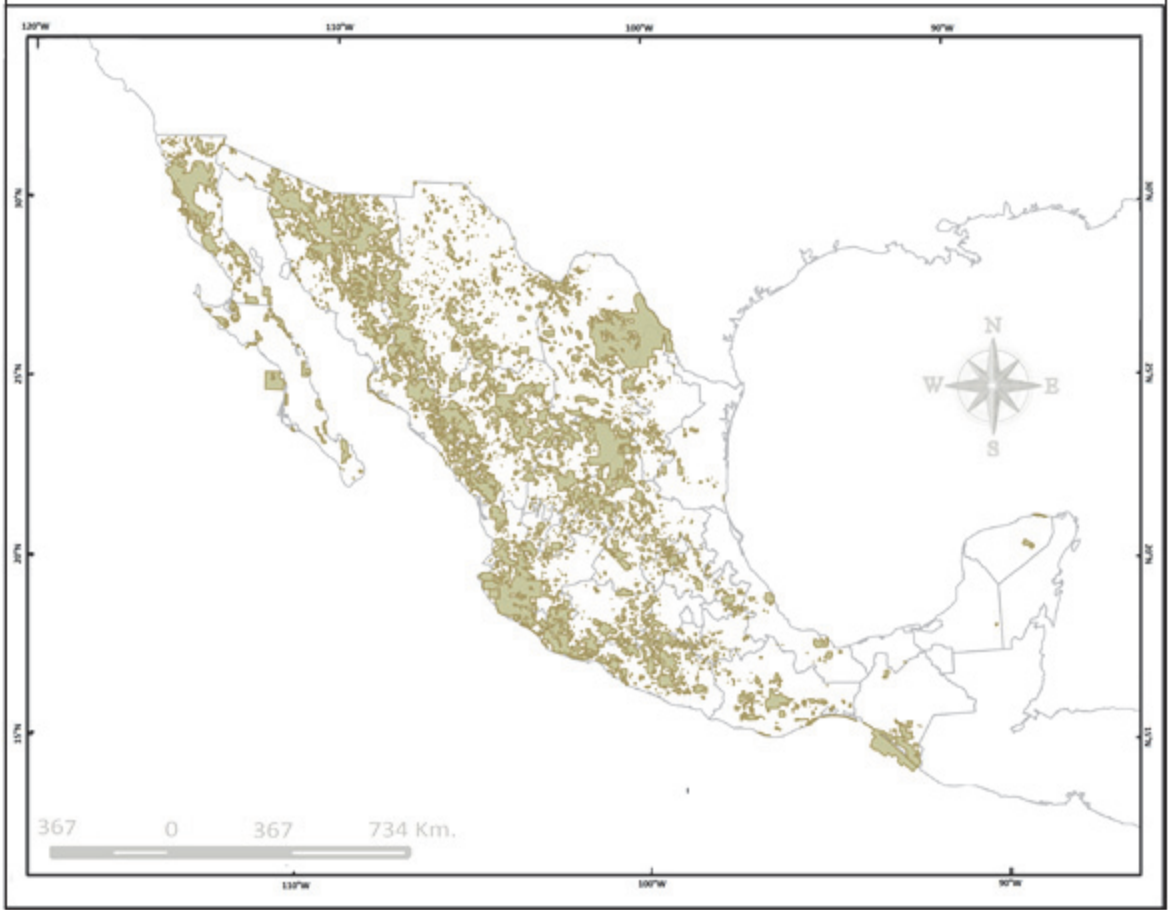

Mapa 4. Concesiones mineras en México.

Fuente: SE, 2014. Mapa concesiones mineras. Digitalización propia.

Por otra parte, existe mucha controversia en torno a la estimación de la superficie concesionada a la minería en México. Hay datos que van desde el 16\% de la superficie Nacional, como lo maneja la Secretaría de Economía (SE) a 70\% según estiman algunas organizaciones sociales como la Red Mexicana de Afectados por la Minería (REMA). El dato aquí presentado es producto de la cartografía generada (véase Mapa 4) y de la revisión de los títulos de concesión minera.

\section{Agua y minería en México}

La minería genera una gran cantidad de daños al medio ambiente, sobre todo a los recursos hídricos, que son el soporte de la gente que vive en las zonas mineras (Bridge, 2004b; Bebbington y Bury, 2009). Al respecto, a continuación comparamos la disponibilidad hídrica en los acuíferos de México con respecto a proyectos mineros.

En el Mapa de Acuíferos y disponibilidad hídrica (véase Mapa 5) nótese cerca del $40 \%$ del total de proyectos coinciden con acuíferos con disponibilidad hídrica. Lo 
cual, si bien es grave en cualquier región, lo es más en regiones áridas o semidesérticas donde, de por sí, el recurso es escaso. Como en el acuífero compartido entre Chihuahua, Sonora y Sinaloa dónde hay más de 80 proyectos mineros. Respecto a los acuíferos con déficit hídrico, en el Mapa 6 se puede observar como prácticamente $90 \%$ de los acuíferos sobre explotados en México coinciden con concesiones mineras.

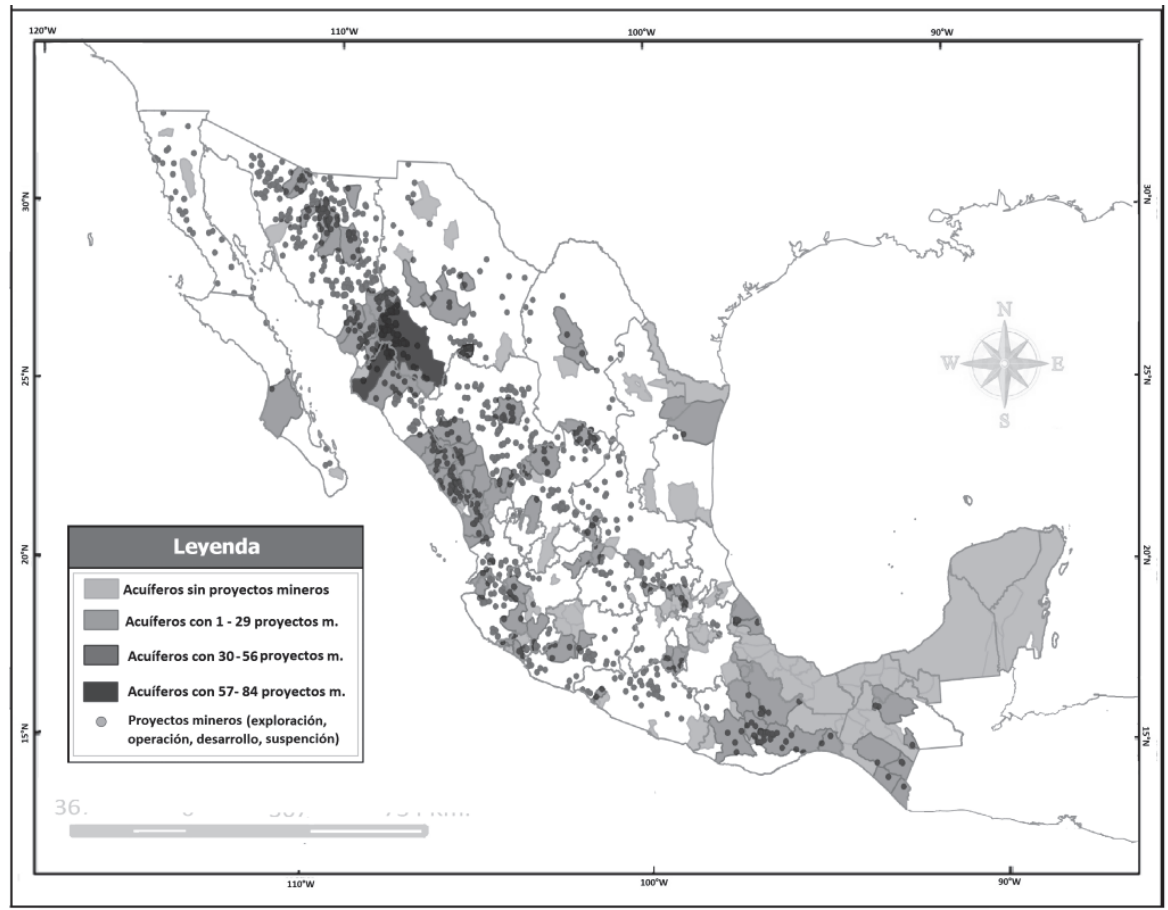

Mapa 5. Acuíferos con disponibilidad hídrica y proyectos mineros en México.

Fuente: elaboración propia en base a: SGM, 2011; SE, 2014; CONAGUA, 2014; MMC, 2014.

Si bien el agua utilizada en la minería es esencialmente subterránea —éstos debido a los grandes volúmenes necesarios-, vale la pena señalar en qué medida los ríos y cuerpos de agua en México coinciden con esta actividad. La relevancia del presente análisis radica en que, un río que pasa por un proyecto minero puede ser — potencialmente - agotado. Pues, la legislación al respecto es muy laxa. La minería, por ejemplo, tiene derecho a aprovechar las aguas preferentemente sobre otras actividades como consumo humano (Ley Minera Art.19. Fracciones V, VI). Además la Ley Federal de Derechos establece en su Artículo 224 que "No se paga- 
rán derechos por las aguas que pasen, broten o aparezcan en el laboreo de las minas". Si una empresa hace un pozo dentro de las concesiones mineras y gasta miles de $\mathrm{m}^{3}$ por segundo, no está obligada a pagar por ello, pues los $\$ 5.00$ que paga por hectárea concesionada al año le dan ese derecho.

Así mismo, cabe señalar que la mayoría de los ríos del occidente del país coinciden con concesiones mineras. Este hecho es preocupante, no solamente por la posibilidad de agotar el agua de dichas corrientes, sino también porque un cuerpo de agua que se encuentra dentro de un proyecto minero corre alto riesgo de ser contaminado. Los derrames en las presas de jales son más comunes de lo que las compañías mineras están dispuestas a aceptar. Un claro ejemplo de ello es el reciente derrame de 40 mil metros cúbicos de tóxicos en el Río Sonora por parte de la Minera Cananea —una de las más importantes del país.

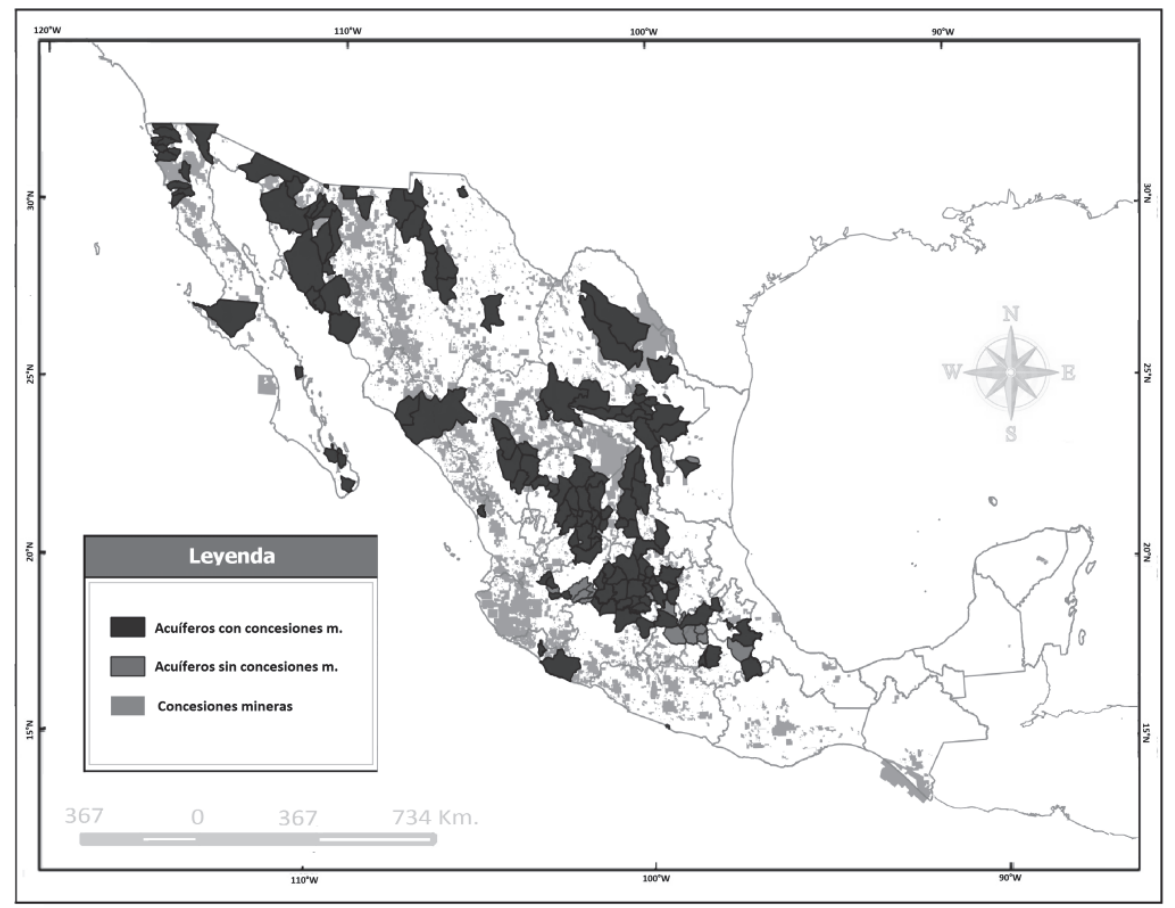

Mapa 6. Acuíferos con déficit hídrico y concesiones mineras en México.

Fuente: elaboración propia en base a: SGM, 2011; SE, 2014; CONAGUA, 2014; MMC, 2014. 


\section{Áreas de conservación ecológica y minería}

Las Áreas Naturales Protegidas (ANP) son porciones del territorio nacional, terrestres o acuáticas, representativas de los diferentes ecosistemas en donde el ambiente original no ha sido modificado en su esencia por la actividad del hombre y que están sujetas a regímenes especiales de protección, conservación, restauración.

Estas áreas están bajo la administración de la Comisión Nacional de Áreas Naturales Protegidas (CONANP). Dicha institución, para el año 2014 reporta que existen 179 áreas naturales protegidas en México. Tal cifra es la suma de todas las categorías en que éstas se dividen (Reservas de la Biosfera, Parques Nacionales, Monumentos Naturales, Áreas de Protección de Recursos Naturales, Santuarios Naturales y Áreas de Protección de Flora y Fauna).

Otras áreas naturales con categoría de conservación ecológica son los sitios RAMSAR. Que se refieren a los humedales inscritos en la convención Ramsar. Un tratado internacional para asegurar la protección de los humedales a nivel internacional, que se firmó en la ciudad de Ramsar, Irán en 1971. Para el año 2012 la CONANP (2013) señaló que existen 138 sitios Ramsar en México.

Además, en 1997 se estableció el Sistema de Unidades de Manejo para la Conservación de la Vida Silvestre (SUMA). Actualmente existen 1761 UMA's en México. Las UMA buscan promover esquemas alternativos de producción compatibles con el cuidado del ambiente, a través del uso racional, ordenado y planificado de los recursos naturales renovables en ellas contenidos, frenando o revirtiendo los procesos de deterioro ambiental.

Si bien es cierto que las reservas ecológicas son un modelo debatible de conservación de la biodiversidad, pues son también territorios contenciosos disputados por actores locales e instituciones federales y generalmente implican despojo o desplazamiento; consideramos que la existencia de éstas áreas es el indicador más accesible para ubicar las zonas con alto grado de biodiversidad o valoradas ecológicamente por algún criterio especial. Por lo tanto, para conocer los efectos de la minería en la biodiversidad de México comparamos la sobre posición de esta actividad con todas las áreas naturales bajo alguna categoría de conservación ecológica, en relación a las concesiones y proyectos mineros en México.

El resultado de dicho análisis es el siguiente, de 2,078 áreas (ANP, Sitios Ramsar y UMA's), 3\% cuenta con proyectos mineros al interior de sus polígonos. Lo cual, pese a que pareciese un porcentaje bajo, no es menospreciable pues ello significa que existen 86 proyectos en regiones que la misma CONABIO reconoce como imprescindibles para la conservación de la biodiversidad en México.

Para el caso de las concesiones mineras en ANP (Mapa 7), el escenario cambia drásticamente, pues ese simple 3\% asciende a $46 \%$, véase Gráfica 4. De las 2078 áreas naturales bajo alguna categoría de conservación ecológica, 958 tienen concesionado alguna porción de su territorio. Todas las del Norte y centro del país prácti- 
camente lo están. Esto es posible ya que el Reglamento de la Ley General del Equilibrio Ecológico y la Protección al Ambiente (LEGEPA) en materia de Áreas Naturales Protegidas no prohíbe realmente la minería dentro de ANP's. En su artículo 87 establece que "De acuerdo con la declaratoria se establecen las siguientes prohibiciones, salvo que se cuente con la autorización respectiva"; entre esas prohibiciones está remover o extraer material mineral. Así, la minería está permitida en ANP siempre y cuando se tenga autorización por parte de la CONANP para realizar obras y trabajos de exploración y explotación mineras.

El resultado de dicha política de favorecimiento a la minería es que, poco más de la mitad de Reservas de la Biosfera en México están concesionadas a la minería, para Parques Nacionales y Monumentos Naturales la cifra es cercana al 20\%, para las Áreas de Protección de Recursos Naturales casi 90\%. Cerca de la mitad de las Áreas de Protección de Fauna y Flora y casi el 30\% de los Santuarios Naturales coinciden también con concesiones mineras. No decimos que es la totalidad de su extensión, pero sí en parte. Al respecto ver Tabla 3.

Tabla 3

Categorías de ANP's y concesiones mineras

\begin{tabular}{lrcc}
\hline \multicolumn{1}{c}{ Categoría ANP } & Número & $\begin{array}{c}\text { Con } \\
\text { concesión }\end{array}$ & $\begin{array}{c}\text { \% respecto al total } \\
\text { ANP's }\end{array}$ \\
\hline Reservas de la Biosfera & 41 & 23 & 56.1 \\
Parques Nacionales & 69 & 12 & 17.4 \\
Monumentos Naturales & 5 & 1 & 20.0 \\
Áreas de Protección de & 8 & 7 & 87.5 \\
Recursos Naturales & & & 47.4 \\
Áreas de Protección de & 38 & 18 & 27.8 \\
Fauna y Flora & & 5 & 37 \\
Santuarios & 18 & 66 & \\
\hline \multicolumn{1}{c}{ Total } & 179 & &
\end{tabular}

Fuente: Elaboración propia en base a cartografía generada.

\section{Minería y pueblos indígenas}

Un tercer tema altamente aludido en el debate público sobre minería es el de la violación a los derechos de los pueblos originarios o indígenas y el acaparamiento de sus territorios (Bebbington, 2009; Bárcenas y Eslava, 2011; Organización de las Naciones Unidas — ONU, 2013; Tetreault, 2014). En el plano nacional, México cuenta con diversos instrumentos que reconocen los derechos de los pueblos indígenas e instrumentos jurídicos que definen la propiedad de la tierra. 


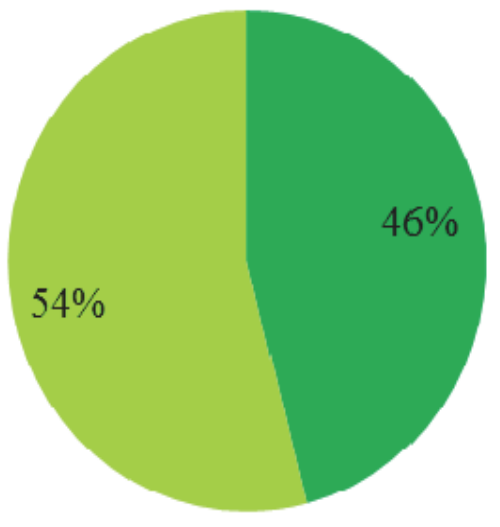

Con concesiones

Sin concesiones

Gráfica 4. Áreas de conservación y concesiones mineras.

Fuente: CONANP 2012, 2013, 2014; SIAM, 2013.

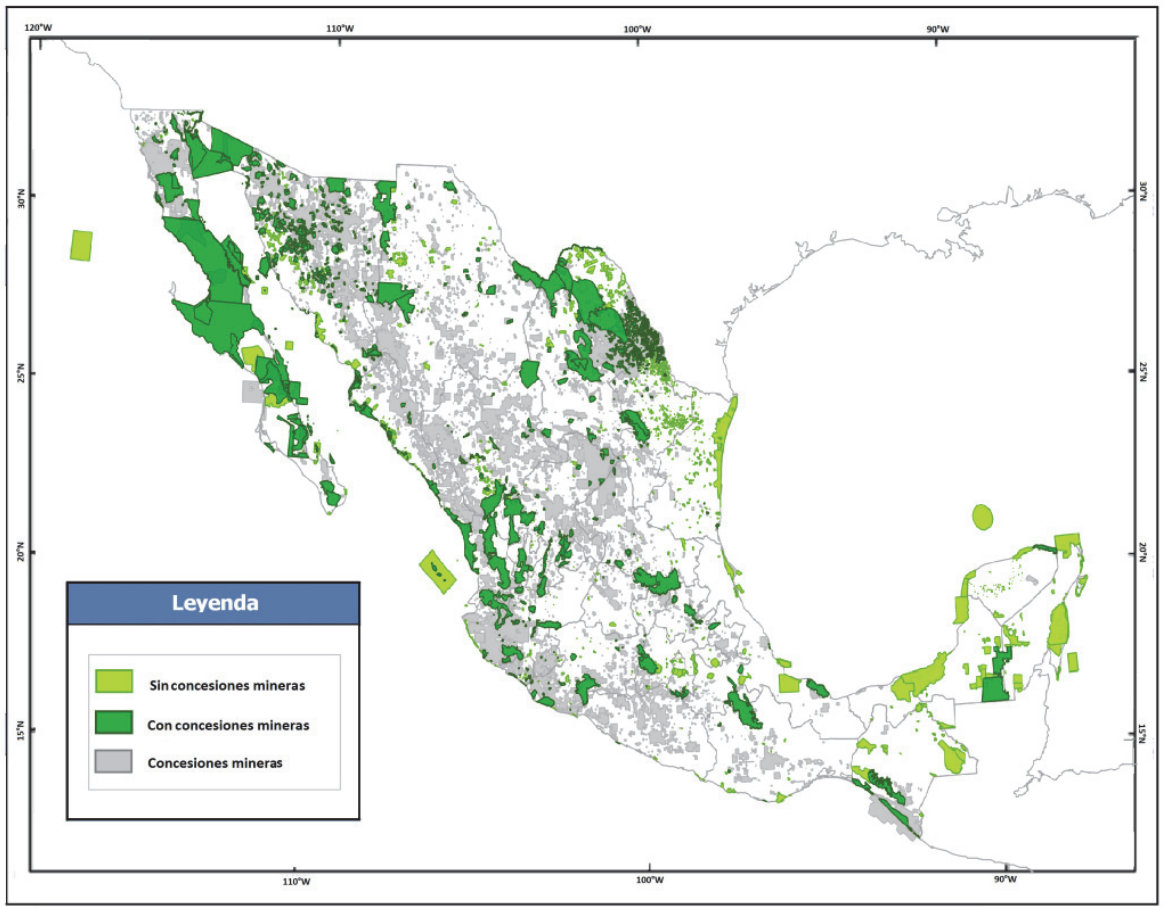

Mapa 7. Áreas de conservación ecológica y concesiones mineras en México. Fuente: CONANP 2014; SIAM, 2013. 


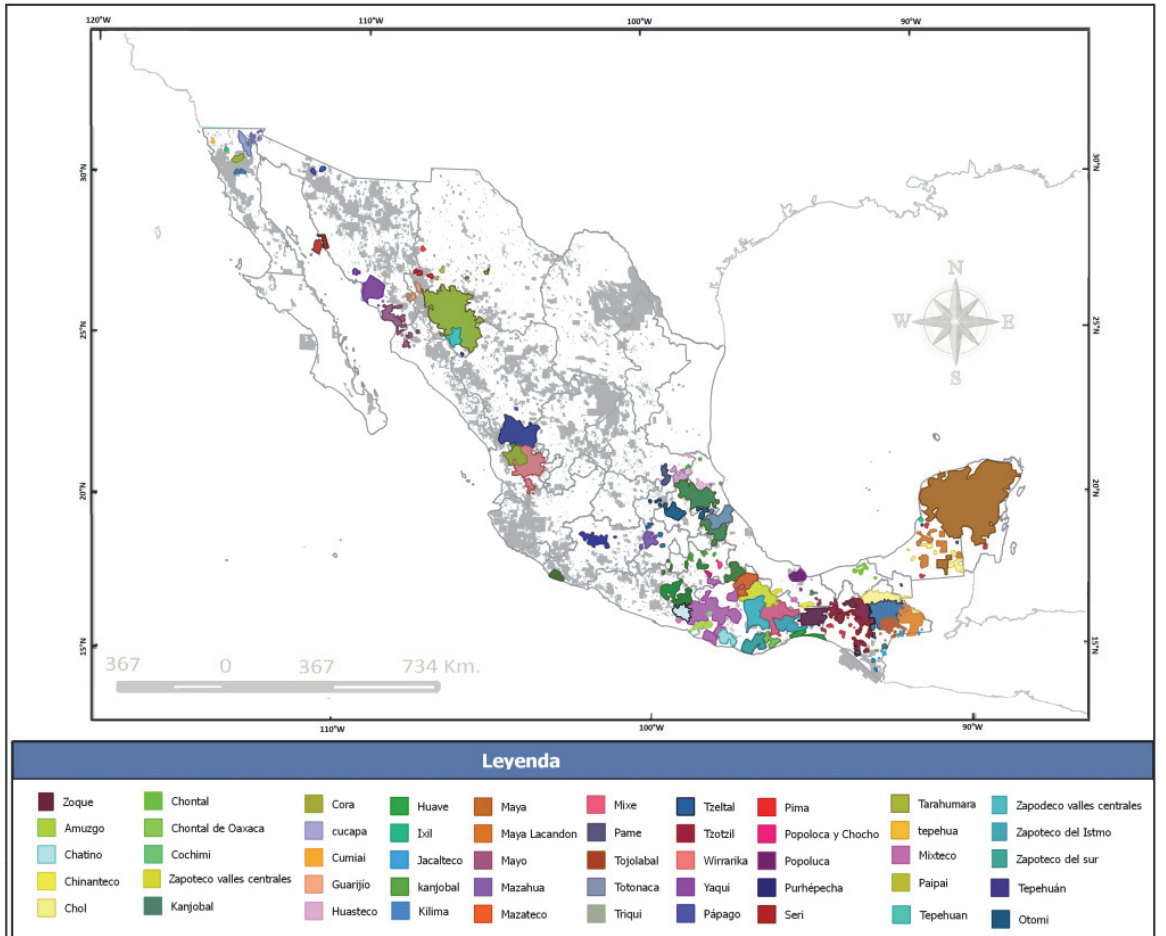

Mapa 8. Territorios actuales de los pueblos indígenas y concesiones mineras en México. Fuente: Boegue, 2011; SIAM, 2014.

La Constitución Política de los Estados Unidos Mexicanos (CPEUM), en su artículo $1^{\circ}$ afirma que "todas las personas gozarán de los derechos humanos reconocidos en esta Constitución y en los tratados internacionales de los que el Estado Mexicano sea parte, así como de las garantías para su protección". El artículo $2^{\circ}$ reconoce el derecho de los pueblos indígenas a la libre determinación, para, entre otros derechos: "acceder, con respeto a las formas y modalidades de propiedad y tenencia de la tierra".

Además, el Convenio 169 de la Organización Internacional del Trabajo (OIT) afirma en su apartado de tierras (artículos 13 al 19), la responsabilidad de los Estados de respetar y proteger las tierras y los territorios de los pueblos indígenas, incluyendo los recursos naturales. En este caso, el artículo 15 establece en su párrafo $1^{\circ}$ que los pueblos indígenas tienen también derecho a participar en la administración y conservación de dichos recursos. En su párrafo 2 señala que:

En caso de que pertenezca al Estado la propiedad de los minerales o de los recursos del subsuelo, o tenga derechos sobre otros recursos existentes en las tierras, los go- 
biernos deberán establecer o mantener procedimientos con miras a consultar a los pueblos interesados, a fin de determinar si los intereses de esos pueblos serían perjudicados y en qué medida, antes de emprender o autorizar cualquier programa de prospección o explotación de los recursos existentes en sus tierras.

Contrario a la legislación sobre el tema, la cartografía generada ejemplifica cómo, al igual que para las áreas naturales, en la práctica la legislación permite la instalación de proyectos mineros en territorios indígenas. Para dicho análisis utilizamos la cartografía generada por Eckart Boegue (2012) para la delimitación de los territorios de los pueblos indígenas contemporáneos. Dicho autor considera territorios de los pueblos indígenas las porciones en donde hoy viven los pueblos indígenas, según los registros del Censo Nacional de Población y Vivienda, 2000 efectuado por el INEGI.

En el Mapa 8 se puede observar cómo cerca del 50\% de los territorios de los pueblos indígenas de México tienen concesiones mineras. Pese a que existe legislación nacional e internacional sobre derechos de los pueblos indígenas en México, en la práctica no se cumple tales disposiciones. Para que se respetara el derecho a la libre determinación de los pueblos de su condición política y su desarrollo económico, social y cultural se requeriría que se reconozca su espacio vital, el territorio. El artículo 26 de la Declaración señala que los pueblos indígenas tienen "derecho a poseer, utilizar, desarrollar y controlar las tierras, territorios y recursos que poseen en razón de la propiedad tradicional u otra forma tradicional de ocupación o utilización”, pero eso en México significaría reconocerles 28,033,093 hectáreas (Boegue, 2011), es decir, $1.5 \%$ del territorio nacional. Y esta no parece ser la intención de ninguno de los gobiernos en turno.

\section{Conclusiones}

Lo desarrollado a lo largo de este texto muestra cómo, debido a una mayor apertura y a cambios en la normatividad minera, se han otorgado mayores facilidades a las empresas extranjeras. Las leyes que regulan la minería ${ }^{1}$ no hacen prohibiciones significativas a la industria. Esta puede establecerse - y lo hace - en zonas importantes para el cuidado del recurso hídrico y la biodiversidad, y para los pueblos indígenas.

\footnotetext{
La legislación que regula la actividad minera en México se encuentra en: la Constitución Política de los Estados Unidos Mexicanos (CPEUM), la Ley Minera, la Ley General de Bienes Nacionales, la Ley Agraria, la Ley General de Equilibrio Ecológico y Protección al Ambiente, la Ley General para la Prevención y Gestión Integral de los Residuos, la Ley de Aguas Nacionales, la Ley de Inversión Extranjera, la Ley Federal de Derechos, la Ley Orgánica de la Administración Pública, el Código Civil, la Ley de Salud, la Ley de Desarrollo Forestal Sustentable y la Ley de Patrimonio Natural y Biodiversidad.
} 
La sistematización, actualización y espacialización de la información más relevante sobre el tema (concesiones, proyectos) nos permitió hacer una breve caracterización del sector minero. Al respecto, hemos demostrado como la minería en México ha transitado hacia la extracción de minerales preciosos (oro y plata). Lo cual cambia el debate público de si es necesaria o no la minería, hacia la discusión de ¿qué minería es necesaria? en qué escala o magnitud y cuáles metales son indispensables para la vida y cuáles los son meramente para la acumulación de capital. Si bajara la "fiebre del oro" podrían, por ejemplo, reducirse en casi un $70 \%$ los proyectos mineros en México.

Otro aporte del trabajo fue el esbozo de la nueva geografía de la minería en México, en términos de su relación con temas clave como el agua, la biodiversidad y los pueblos indígenas. Fue difícil encontrar patrones espaciales significativos, pues dicha actividad se establece a lo largo y ancho del territorio nacional. En ese sentido, la cartografía generada muestra la "territorialidad del capital", una territorialidad eficientista que se basa en la concepción del territorio como aquel que debe pensarse en pro de beneficios particulares, que se pueda integrar a la dinámica económica global. Como espacio a ser utilizado, explotado y dominado para la acumulación de capital (Svampa, 2008), a pesar de los usos sociales que este tenga. Bajo esta noción se valoriza la acumulación de capital a costa de todo.

Así mismo, los mapas aquí presentados ejemplifican como el sistema político mexicano está corrompido y es servil a los intereses de las grandes corporaciones a costa de los derechos humanos más básicos de la población local (al agua, al medio ambiente sano, la identidad cultural, una vida digna, etc.). Lo cual evidencia también el doble discurso del Estado que, por un lado tiene políticas conservacionistas y restrictivas de la biodiversidad y por otro, en congruencia con el modelo neoliberal, permite que las compañías mineras se establezcan en, literalmente, cualquier sitio.

En ese sentido, es importante rescatar la relevancia de los sistemas de información geográfica como herramienta para contribuir a análisis de índole social y al mismo tiempo, proveer información cartográfica a la ciudadanía, que de otro modo, no tendría acceso a ella. Lo cual es en suma valioso pues, consideramos, una sociedad bien informada tendrá las herramientas necesarias para una mejor toma de decisiones encaminadas al bien común.

\section{Bibliografía}

Bárcenas, F. y Eslava, M. (2011). El mineral o la vida, Pez en el árbol, México. Bebbington, A. (2009). "Actores y Ambientalismo: continuidades y cambios en los conflictos socioambientales por minería en Perú", en Minería y Territorio en el Perú. Conflicto, resistencias y propuestas en tiempo de globalización, Programa 
de Democracia y Ecuador Debate/ Tema central 125 Transformación Global, Conacami, Cooperación, Universidad Mayor de San Marcos, Lima.

Boegue, E. (2012). Patrimonio biocultural de los pueblos indígenas de México, Instituto Nacional de Antropología e Historia, Comisión Nacional para el Desarrollo de los Pueblos Indígenas, México.

Bridge, M. (2004). "Mapping the Bonanza: Geographies of Mining Investment in an Era of Neoliberal Reform", Professional Geographer, vol. 56, núm. 3, pp. 406-421.

CONAgua (2014). Disponibilidad de agua por acuífero. Tablas por entidad federativa, México.

CONAMP (2014). Áreas Naturales Protegidas en México.

FUNDAR (2013). "Informe sobre industrias extractivas", Centro de Análisis e Investigación, México.

Garibay, C.; Boni, A.; Pánico, F.; Urquijo, P. y Klooster, D. (2011). “Unequal Partners, Unequal Exchange: Goldcorp, the Mexican State, and Campesino Dispossession at the Peñasquito Goldmine", Journal of Latin American Geography, vol. 10 no. 2, Conference of Latin Americanist Geographers, pp. 153-176.

Giraud, M. (2012). Algunos aspectos Ambientales, económicos, legales y sociales de la Minería, Provincial del Ambiente ante la Comisión Evaluadora Interdisciplinaria Ambiental Minera de Mendoza, Universidad Nacional de Cuyo, Mendoza, Argentina.

Gonzáles, L. (2012). “Conflictos entre concesionarios mineros y comunidades. La lógica del estado ausente en los procesos de mediación y la estrategia de resistencia de las comunidades indígenas de Guerrero", ponencia presentada en el $2^{\circ}$ Congreso Internacional PRE-ALASRU, septiembre, Cuernavaca, Morelos, México.

Gudynas, E. (2011). “Caminos para las transiciones post extractivistas” en Alayza, A. y Gudynas, E. (eds.), Transiciones, post-extractivismo y alternativas al extractivismo en el Perú, Centro Latino Americano de Ecología Social (CLAES), Lima.

Mexico Mining Center (MMC), 2014, <http://www.mexicominingcenter.com/ web/> (15-02-2014).

Mudd, G. (2007). "Global Trends and Gold Mining: Toward Quantifying Environmental and Resource Sustainability", Resources Policy, no. 32, pp. 42-56.

O'Faircheallaigh, C. (2010). "Aboriginal-Mining Company Contractual Agreements in Australia and Canada: Implications for Political Autonomy and Community Development", Canadian Journal of Development Studies, vol. XXX, nos. 1-2, pp. 69-86.

Observatorio Latinoamericano de Conflictos Mineros (OCMAL), 2013, $<$ www.conflictosmineros.net> (20-01-2014). 
Organización de las Naciones Unidas (ONU), (2013) "Estudio sobre las industrias extractivas en México y la situación de los pueblos indígenas en los territorios en que están ubicadas estas industrias", Foro Permanente para las Cuestiones Indígenas, $12^{\circ}$ período de sesiones, Nueva York, 20-31 mayo.

Peláez, J. (2013). "La campaña nacional por una nueva Ley minera”, ponencia presentada en el contexto de las Jornadas mundiales contra la minería Universidad Autónoma de la Ciudad de México (UACM), Universidad Nacional Autónoma de México (UNAM), noviembre, México.

Pérez-Jiménez, S. (2012). Aproximación geográfica a los conflictos territoriales y ambientales en México: La lucha por los recursos naturales a partir del caso de la minería. tesis de la licenciatura en Ciencias Ambientales, Universidad Nacional Autónoma de México (UNAM), México.

Secretaría de Economía (SE), (2013). Participación de México en la producción minera mundial del 2012, Dirección de Control Documental e Indicadores Estratégicos, México.

(2014). Proyectos mineros de capital extranjero. Dirección General de Desarrollo Minero Disponible en: <http://portalweb.sgm.gob.mx/economia/ es/mineria-en-mexico/lista-de-proyectos.html>

Servicio Geológico Mexicano (SGM), (2011). Panoramas Mineros por Estado, Secretaría de Economía, Coordinación General de Minería, México.

SIAM (2014), Sistema de Administración Minera, Secretaría de Economía.

Svampa, M. (2008). "La disputa por el desarrollo: territorio, movimientos de carácter socio-ambiental y discursos dominantes" ponencia presentada en el Seminario "Interrogating the Civil Society Agenda", Universidad de Massachussets, Amhers, abril.

Tetreault, D. (2012). "The Political Ecology of Mining in Mexico", IDS Working Paper, St. Mary’s University, Texas.

TTC (2010). TTC Corporate Policy - Open Data. Toronto Transit Commission Report No. 31, Toronto, Canada.

Wallenius, C. (2010). "Minería y respuestas sociales en Mezcala, Guerrero: un análisis desde la geopolítica local", en Delgado-Ramos (coord.), Ecología política de la minería en América Latina, UNAM, México, pp. 251-280. 